\title{
SUPPORTED Co CATALYSTS PREPARED AS THIN FILMS BY MAGNETRON SPUTTERING FOR SODIUM BOROHYDRIDE AND AMMONIA BORANE HYDROLYSIS
}

M. Paladini ${ }^{1}$, G. M. Arzac ${ }^{1}$, V. Godinho ${ }^{1}$, M.C. Jiménez De Haro ${ }^{1}$, A. Fernández ${ }^{1}$

${ }^{1}$ Instituto de Ciencia de Materiales de Sevilla, CSIC-Univ. Sevilla, Américo Vespucio 49, Isla de la Cartuja, Seville, Spain.

" Corresponding author: phone number: +34954489552 , fax number: +34954460665 , e-mail: gisela@icmse.csic.es

\begin{abstract}
Supported Co catalysts were prepared for sodium borohydride and ammonia borane hydrolysis by magnetron sputtering for the first time under different conditions. Ni foam was selected as support. Deposition conditions (time, pressure, and power) were varied to improve catalytic activity. A decrease in deposition power from 200 to $50 \mathrm{~W}$, leads to a decrease in crystallite and column size and a higher activity of catalysts. The increase in deposition pressure from $1.5 \times 10^{-2}$ to $4.5 \times 10^{-2}$ mbar produces same effect but in this case the enhancement in activity is higher because amorphous materials were obtained. The highest activity for SB hydrolysis was $2650 \mathrm{ml}^{-\mathrm{min}^{-1}}$. $\mathrm{g}_{\mathrm{cat}}{ }^{-1}$ for the $50 \mathrm{~W}$ Co 4.5 (4h) sample $\left(E_{\mathrm{a}}=60 \pm 2 \mathrm{~kJ} \mathrm{~mol}^{-1}\right)$. For AB hydrolysis activity for the 50W Co 3.2 (4h) sample was similar. Durability of the thin films was tested for both reactions upon cycling (14 cycles). Diluted acid washing was effective to recover the activity for sodium borohydride reaction but not for ammonia borane hydrolysis. The strong $\mathrm{Co}-\mathrm{NH}_{3}$ interactions explain the non-efficiency of the acid washing.
\end{abstract}

\section{Keywords}

sodium borohydride; catalytic hydrolysis; Co thin films; magnetron sputtering; durability

\section{Introduction}

Nowadays, hydrogen is considered as a potential future energy carrier owing to its high energy content, renewability and non-polluting energy release (reaction with oxygen produces water as only by-product). However, one bottleneck for the development of a hydrogen economy is the issue of hydrogen storage. Over the last 
decade hydrolysis of chemical hydrogen storage materials such as sodium borohydride $(\mathrm{SB})$ and ammonia borane $(\mathrm{AB})$ have proven to be an efficient approach for hydrogen generation [1-4]. Both hydrides are stable in dry air and easy to handle and combine low molecular weight (37.8 and 30.7 g. $\mathrm{mol}^{-1}$ for SB and $\mathrm{AB}$ respectively) with high gravimetric hydrogen content, making them very attractive. Catalyzed sodium borohydride and ammonia borane hydrolysis are safe exothermic reactions that produce hydrogen according to (1) and (2) respectively [1-4].

$$
\begin{aligned}
& \mathrm{NaBH}_{4}+2 \mathrm{H}_{2} \mathrm{O} \rightarrow 4 \mathrm{H}_{2}+\mathrm{NaBO}_{2} \\
& \mathrm{NH}_{3} \mathrm{BH}_{3}+2 \mathrm{H}_{2} \mathrm{O} \rightarrow \mathrm{NH}_{4}^{+}+\mathrm{BO}_{2}^{-}+3 \mathrm{H}_{2}
\end{aligned}
$$

Both spontaneous reactions are slow and need catalysts to occur at appreciable rates. One of the main obstacles for the practical application of the catalytic hydrolysis of these ( $\mathrm{SB}$ and $\mathrm{AB}$ ) chemical hydrogen storage materials is the need to develop efficient, economical and durable catalysts to accelerate reactions (1) and (2) [1-4]. Co based catalysts have demonstrated in the past decade to be the most advantageous and cost effective for reaction (1) [1-3,5-7] and also for reaction (2) [4-7].

From a technological and operational point of view, i.e. system and reactor design, it is highly desirable to have Co catalysts in a supported form [2-3]. Supported catalysts do not aggregate as powder catalysts and can be recovered from the reaction medium, to be reused in multi-use applications. Traditionally, Co supported catalysts have been mainly prepared by the impregnation-chemical reduction method [1-14]. In most cases, sodium borohydride is employed as reducing agent leading to the formation of Co-B materials [15-18].

Electrodeposition has also been presented as a convenient method for scale-up the production of supported Co catalysts [19-20]. However for non-conducting substrates an intermediate step for the deposition of a conductive layer is need.

Pulsed Laser Deposition is a PVD (Physical Vapor Deposition) technique that has been employed for the preparation of catalysts for SB and AB hydrolysis. Co-B thin films and more recently, Co nanoparticles on a B film have been prepared by this method [21-24]. This technique allows to deposit high density granular films of different roughness with the target stoichiometry. [21-25]

Magnetron sputtering is a PVD technique that allows the deposition of thin films with precise controlled thickness over a wide variety of substrates due to the low deposition temperature of the process. It can be easily scaled to industrial processes and is a low cost process without the need of precursors avoiding emissions of toxic by- 
products. The control of the deposition conditions allows controlling the microstructure of the coatings and sputtering conditions can be readily reproduced from run-to-run. This technique has proven before to be a versatile tool to prepare nanostructured and porous materials in our group [26-28]. To our knowledge, magnetron sputtering has not been reported as preparation technique for supported Co catalysts for sodium borohydride or ammonia borane hydrolysis before [29].

In this paper, $\mathrm{Ni}$ foam supported nanostructured Co coatings $(\mathrm{Co} / \mathrm{Ni}$ foam) were prepared as thin films by magnetron sputtering in a wide range of conditions as catalysts for reactions (1) and (2). As a proof of concept, PTFE membranes were also used as thin film support.

Catalytic activity of the $\mathrm{Co} / \mathrm{Ni}$ foam materials was tested for reactions (1) and (2) in $4.5 \mathrm{wt} \% \mathrm{NaOH}$ and pure water respectively. Activity is correlated to deposition conditions, crystallinity and nanostructure. Durability was also tested for both reactions upon cycling (14 cycles) and compared. A reactivation step consisting in dilute acid washing was also employed and the results discussed.

\section{Experimental Section}

\subsection{Catalysts preparation}

To investigate the influence of the different deposition parameters on the activity of the supported catalyst, Co nanostructured coatings were deposited under different pressure and dc power. Table 1 summarizes the deposition conditions employed.

The coatings were deposited using a 2" magnetron from AJA in magnetic target configuration, from a pure cobalt target (Kurt J. Lesker 99.95\% pure, $1 \mathrm{~mm}$ thick) under pure argon atmosphere. The base pressure before deposition was $10^{-6} \mathrm{mbar}$, and working pressures from $1.5 \times 10^{-2}$ to $4.5 \times 10^{-2}$ mbar of Ar were employed to prepare the coatings.

The influence of the deposition power on the microstructure and consequently on activity was investigated by changing the dc power supplied from 50 to $200 \mathrm{~W}$.

The coatings were deposited directly on commercial Ni foam (Goodfellow 1.6 $\mathrm{mm}$ thick, 95\% porosity, 20 pores $/ \mathrm{cm}$ ) and PTFE (Polytetrafluoroethylene) membranes (Whatman $0.2 \mu \mathrm{m}$ pore size, Polypropylene backed). Before deposition, Ni foam or PTFE membranes were cut into $0.5 \times 0.5 \mathrm{~cm}^{2}$ pieces and grouped to be used in a small reactor (see section 2.3). Each group was weighted before and after each deposition to 
obtain the total mass of catalyst deposited. Depositions times of 2 and 4 hours allowed to obtain different catalyst mass under similar deposition conditions.

$\mathrm{Ni}$ foam was washed first with ethanol and then with $\mathrm{HCl} 0.1 \mathrm{M}$ in an ultrasonic bath before each synthesis. No previous treatment was done on PTFE membranes. As a reference for characterization (specially Electron Microscopy), in each deposition Si (100) substrates were included. The Si substrates were cleaned with acetone and dried in a nitrogen flow.

Catalysts are named by their deposition/synthesis conditions and the support. As an example, 200W Co 1.5 (2h) catalyst is the Ni foam supported catalyst prepared by magnetron sputtering at $200 \mathrm{~W}$ power and $1.5 .10^{-2} \mathrm{mbar}$ Ar pressure during $2 \mathrm{~h}$. To avoid confusion, Table 1S (included as supporting information) correlates catalyst name and preparation conditions. In those cases in which the support is different from $\mathrm{Ni}$ foam, it will be clearly indicated in the text.

\subsection{Catalysts characterization}

Scanning electron microscopy (SEM) was performed to study the morphology of the coatings on the different substrates and the thickness of samples deposited on $\mathrm{Si}$ (100) in a high resolution FEG microscope, HITACHI S4800 operating at $2 \mathrm{kV}$ equipped with an EDX detector (Bruker X-Flash 4010). The microstructure of the coatings was investigated by Transmission Electron Microscopy (TEM), performed using a Philips CM 200 microscope with 2.4 Å resolution. Cross-sectional transmission electron microscope (XTEM) specimens were prepared in the conventional way by mechanical polishing followed by $\mathrm{Ar}^{+}$ion milling to electron transparency of coatings deposited on Si substrates. Surface topography was investigated by atomic force microscopy (AFM) Nanotec system working in tapping mode, with Si tips of $150 \mathrm{kHz}$ resonant frequency and $20 \mathrm{~N} \mathrm{~m}^{-1}$ spring constant. 3D surface images of $5 \times 5 \mu \mathrm{m}$ were acquired and analyzed with the WSxM free available software from Nanotec [30].

$\mathrm{X}$-ray diffraction measurements were performed using $\mathrm{Cu} \mathrm{K} \alpha$ radiation in a Siemens D5000 diffractometer in a Bragg-Brentano configuration in the $2 \theta$ angle range of 20-90 degrees. The surface chemical composition of the catalyst before and after test was investigated by XPS. XPS spectra were recorded with a Leybold Heraus LH electron spectrometer using Al Ka radiation with $40 \mathrm{eV}$ pass energy at normal emission take off angle. The spectra were calibrated with the position $\mathrm{C}$ (1s) (from contamination) signal at $284.9 \mathrm{eV}$. 


\subsection{Hydrogen Generation (HG) test.}

Ni foam supported Co catalysts were tested as following: a certain amount of supported catalyst (1-8 mg) was placed at the bottom of a three necked heart-shaped flask. The flask was immersed in a water bath maintained at $23 \pm 0.5^{\circ} \mathrm{C}$ and connected to a $100 \mathrm{ml}$ gas burette. The reactions 1 and 2 started by injecting $38 \mathrm{mg}$ of SBH dissolved in $1 \mathrm{ml}$ of $4.5 \mathrm{wt} \% \mathrm{NaOH}$ solution or $40 \mathrm{mg}$ of $\mathrm{AB}$ dissolved in $1 \mathrm{ml}$ of MilliQ ${ }^{\circledR}$ water respectively. The amount of generated hydrogen was measured by reading the displacement of the piston in the gas burette (gas-tight by a mercury o-ring) as a function of time. For $\mathrm{AB}$ hydrolysis experiments the produced gas was passed through a flask with $0.1 \mathrm{M} \mathrm{H}_{2} \mathrm{SO}_{4}$ solution to remove ammonia, before directing to the gas burette. No stirring was used for the experiments, except for the stirring effect of the evolved hydrogen. The HGR (Hydrogen Generation Rate, ml.min ${ }^{-1}$ ) was obtained from the slope of the plot of the volume of hydrogen evolved vs. time in linear regime. Experiments were done in duplicate, and showed to be reproducible. In this paper, catalytic activity (expressed in $\mathrm{ml} \cdot \mathrm{min}^{-1} \cdot \mathrm{g}_{\text {catatyst }}{ }^{-1}$ ) is obtained as the slope from the plot of HGR ( $\left.\mathrm{ml} \cdot \mathrm{min}^{-1}\right)$ as a function of the mass of supported catalyst .

To test durability, the above mentioned tests for reaction 1 and 2 were repeated for 14 times. After each test, catalyst was extracted from the reaction medium and washed with distilled water and ethanol then it was dried under a $\mathrm{N}_{2}$ flow. When indicated, the used catalyst was washed with $10^{-4} \mathrm{M} \mathrm{HCl}$. This acid washing is intended to eliminate the adsorbed $\mathrm{BO}_{2}^{-}$ions which produce catalyst deactivation. [20,31] Catalyst loss was evaluated after each test by weighting.

\section{Results and Discussion.}

\subsection{The supported catalyst}

By choosing the adequate deposition conditions, magnetron sputtered highly columnar coatings, with high surface area to be used as catalysts, can be obtained.

Figure 1 shows the morphology and microstructure of the supported Co coatings deposited on $\mathrm{Ni}$ foam and silicon at 200 and 50W, under Argon pressure of $1.5 \times 10^{-2}$ mbar during 2 hours.

In both cases the coatings present columnar growth, typical of magnetron sputtered coatings deposited under low adatom mobility conditions where surface shadowing governs the film growth [32] The cross-sectional SEM and TEM micrographs (Figs 
1.a.1, 1.a.3. and 1.b.1, 1.b.3) reveal that these meso-columns are composed by nanocolumns in a feather-like structure where high intra and inter-columnar porosity can be observed. TEM shows that inside the columns there is a superlattice structure perpendicular to the growth direction, corresponding to nano-columns growing tilted with respect to the growing direction. The width of the meso-columns (average values from SEM in the meso-columns highest dimension) varies from 50 to $350 \mathrm{~nm}$ in the case of the $50 \mathrm{~W}$ coatings and from 150 to $900 \mathrm{~nm}$ when $200 \mathrm{~W}$ dc power is applied. The surface topography of these samples was also investigated by AFM and is shown on Figures 1a.4 and 1.b.4. Clear differences between the two power conditions can be observed, the sample deposited at $50 \mathrm{~W}$ is composed by thinner meso-columns with sharper ends than in the case of the $200 \mathrm{~W}$ catalyst as it can be observed by the AFM profiles. The meso-columns ends present different heights as displayed on the histograms of topography vs number of columns. For $50 \mathrm{~W}$ Co catalyst the majority of meso-columns have a height of $150 \mathrm{~nm}$ while for the $200 \mathrm{~W}$ it is around $225 \mathrm{~nm}$.

The influence of different substrates on the coatings morphology and microstructure was investigated. Figure 2 presents as an example a cobalt coating deposited at $200 \mathrm{~W}$, on $\mathrm{Si}(100)$ in comparison with the same coating deposited simultaneously on nickel foam and PFTE membrane, the former a traditional support for SBH hydrolysis. Magnetron sputtering technique has proven to be able to cover efficiently these supports and conformal coatings with similar columnar structure were obtained. (On supporting information more details on bare and covered $\mathrm{Ni}$ foam and PFTE membrane can be found in Figure 1S.) The higher magnification SEM micrographs (Figure $2 \mathrm{~d}$ ) to f)) show that the columnar microstructure of the catalyst is similar on the different substrates.

\subsection{Catalytic activity of Co coatings for SB hydrolysis}

Activity for SB hydrolysis was measured in $4.5 \mathrm{wt} \% \mathrm{NaOH}$ solution for each prepared Ni foam supported catalyst. Results are summarized in Table 2. As an example, in supporting information (Figure $2 \mathrm{~S}$ ) hydrogen evolution curves and hydrogen generation rate plot as a function of the mass of catalyst are shown for the $50 \mathrm{~W}$ Co 3.2 (4h) sample. For all tested catalysts, hydrogen evolution curves follow a straight line indicating zero-order kinetics in SB concentration. No induction period was detected in 
hydrogen evolution experiments. Thin films have shown to be stable and well adhered to the support in reaction conditions. No catalyst loss was found during hydrolysis tests. The following sections discuss the influence of deposition power, time, and pressure on the microstructure and catalytic activity of Co coatings for SB hydrolysis.

\subsubsection{Effect of Deposition Power}

Table 2 presents the corresponding catalytic activities for deposition powers of 50 and 200W. A significant increase on activity (from 360 to $700 \mathrm{ml} \cdot \mathrm{min}^{-1} \cdot \mathrm{g}_{\text {catalyst }}{ }^{-1}$ ) is observed when deposition power decreases at $1.5 .10^{-2}$ mbar and $2 \mathrm{~h}$ (entries $2 \mathrm{a}$ and $2 \mathrm{~d}$ ). The morphological changes between coatings deposited at different powers, presented in Figure 1 contribute greatly to the different activity. Column width increases with deposition power reducing the number of columns per square nanometer. The study of the effect of deposition power on supported catalysts' surface area and the number of active sites is difficult to study by BET and chemisorption measurements that require high amount of catalyst. Nevertheless, the AFM profiles on Figure 1 show clearly a higher surface area in the case of the $50 \mathrm{~W}$ prepared catalyst.

On the other hand, it is known that amorphous (or nanocrystalline) Co catalysts are more catalytically active than the corresponding crystalline samples [5]. Figure 3 shows the XRD diffractograms of the 50W and 200W PTFE supported cobalt catalysts. Both catalysts show peaks which can be assigned to the hep Co phase. Scherrer analysis on both diffractograms indicates crystal sizes ((100) hcp Co) of $11 \mathrm{~nm}$ and 31 $\mathrm{nm}$ for the 50 and 200W Co catalysts respectively. In this range of conditions catalytic activity can be correlated to the inverse of crystal size. In fact crystal size and columns width both indicate a higher active surface area for the same amount of catalyst when decreasing deposition power. The SAED patterns (Figures 1.a.3 and 1.b.3, inset) indicates more crystallinity in the case of the catalyst deposited at $200 \mathrm{~W}$ in accordance to XRD.

\subsubsection{Effect of deposition time.}

A direct way to control the amount of supported catalyst under the same deposition conditions is by varying deposition time. However it is known that magnetron sputtered columnar films grown under low atom mobility conditions often present $\mathrm{V}$-shaped columns with competitive growth, that become wider from the substrate to the surface. A considerable increase of column width with consequent 
decrease of inter-columnar pores would decrease total surface an influence the catalytic activity of the coatings. Table 1 shows the influence of deposition time for two different powers on film thickness and deposition rate and Figure 4 gives an example of morphology changes from 2 to 4 hours of deposition.

Deposition rate, calculated from SEM cross-sectional thickness measurements, is not affected by deposition time, increasing with the increase of power supplied (0.12$0.13 \mathrm{~nm} / \mathrm{s}$ for $50 \mathrm{~W}$ power and $0.51-0.56$ for $200 \mathrm{~W}$ ). Figure 4 shows a decrease of porosity and an increase of competitive columnar growth for coatings deposited at the same power and double time. The open spaces between columns observed for $2 \mathrm{~h}$ deposition (Fig. 4.a) are occupied by competition of smaller columns for 4h (Fig 4.b). Despite the microstructural differences between both catalysts, no significant effect was found in catalytic activity for sodium borohydride hydrolysis (Table 2, entries 2.a and 2.b). Similar results were found for $50 \mathrm{~W}$ Co 1.5 (2h, 4h) catalysts (Table 2, entries 2.d and 2.e respectively). It seems that the significant increase of smaller meso-columns with feather like structure is enough to increase the total surface area, compensating loss of inter-columnar active places for the catalytic reaction.

\subsubsection{Effect of Deposition Pressure}

Deposition pressure is also a well-known parameter influencing film's microstructure during magnetron sputtering growth of thin films. As a general behaviour the increase of deposition pressure leads to the formation of granulated films that could end in a powder-like film. The effect of deposition pressure in catalytic activity and microstructure was also studied in this work. Supported Co catalysts were deposited at $50 \mathrm{~W}$ during $4 \mathrm{~h}$ on $\mathrm{Ni}$ foam. Deposition pressure was varied in the range of $1.5-4.5$. $10^{-2}$ mbar. Figure 5.a presents details of the morphological changes that occur with the increase of deposition pressure. At low pressure $\left(1.5 \times 10^{-2} \mathrm{mbar}\right)$ the meso-columns (sizes ranging from 50 to $350 \mathrm{~nm}$ width) are composed by nano-columns with a feather like structure with big inter-columnar spaces. As the pressure increases to $2.8 \times 10^{-2} \mathrm{mbar}$ the meso-columns tend to gather in groups forming bigger units where the intercolumnar space is reduced. These units can be considered itselves as different meso-columns with sizes from 70 to $430 \mathrm{~nm}$. A further increase in pressure, to $3.2 \times$ $10^{-2}$ mbar changes the meso-columns structure from feather like to vertically aligned nano-columns. Meso-columns sizes from 20 to $120 \mathrm{~nm}$ can be observed by SEM. At 
$4.5 \times 10^{-2}$ mbar the meso-columns became thinner, $10-70 \mathrm{~nm}$ width. This shows that column size decreases strongly with deposition power.

Catalytic activity as a function of deposition pressure for the $50 \mathrm{~W}$ Co (4h) material is shown in Table 2 (entry 2.e). Figure $5 \mathrm{~b}$ also plots the catalytic activity as a function of deposition pressure. Up to $2 \times 10^{-2}$ mbar, catalytic efficiency does not vary with pressure. Further pressure increase produces a linear increase in activity up to $3.2 \times 10^{-2}$ mbar. Activity falls from the linear trend for higher deposition pressure. Figure 5.b. also plots the average meso-column size as a function of deposition pressure. There is a clear correlation between deposition pressure, column size and catalytic activity. The decrease in column size (more disperse material) observed with deposition pressure directly correlates with activity.

Figure 5.b. shows the XRD diffractograms of the thin films obtained as a function of pressure. The increase of deposition pressure has also an important effect on the crystallite size, since more amorphous and disperse coatings are obtained at higher pressures contributing also to the increase of catalytic activity. Similar dependency of crystallite size on deposition pressure was observed before in literature for Co-based coatings deposited by magnetron sputtering [33].

Increasing deposition pressure has demonstrated to be an efficient strategy to obtain amorphous materials (Fig. 5.b) which exhibit enhanced catalytic activity in respect to those obtained by varying deposition power (section 3.2.1., Fig. 3), with high crystallinity. This shows that the amorphous/nanocrystalline microstructure is a requirement for Co thin film catalysts to obtain higher activity.

The Arrhenius plot for 50W Co 4.5 (4h) catalyst, which exhibited the highest activity, is shown in Fig. 3.S (as supporting information). The resulting activation energy $\left(E_{a}\right)$ was $(60 \pm 2) \mathrm{kJ} \mathrm{mol}^{-1}$ which is lower than the previously reported for bulk cobalt $\left(75 \mathrm{kj}^{\mathrm{mol}}{ }^{-1}\right)$ [34]. For the same prepared thin film, the effect of $\mathrm{NaOH}$ concentration on catalytic activity was also studied. Kinetic tests were performed at 0 , 4.5, 10, $20 \mathrm{wt} \% \mathrm{NaOH}$ and results are shown in Fig. 4.S (as supporting information). The plot of the normalized HGR as a function of $\mathrm{NaOH}$ concentration shows that activity reaches a maximum for $4.5 \mathrm{wt} \% \mathrm{NaOH}$ and then decreases slowly for higher $\mathrm{NaOH}$ concentration. This trend, reported previously for Co and Co-B catalysts, is consistent with the mechanism proposed by Holbrook and Twist, which involves a noncatalyzed intermediate reaction which is dependent on $\mathrm{NaOH}$ concentration [12, 35-36]. 


\subsection{Catalytic activity for $A B$ hydrolysis}

For the 50W Co 3.2 (4h) sample, activity was also measured for AB hydrolysis in pure water. Figure 6.a shows the hydrogen evolution curves. Hydrogen evolution follows a straight line indicating zero-order in $\mathrm{AB}$ concentration with a 2-5 min induction time which decreases with the mass of catalyst. Activity was calculated from de HGR vs mass plot as $2500 \mathrm{ml} \cdot \mathrm{min}^{-1}$. $\mathrm{g}_{\text {cat }}{ }^{-1}$. For the same thin film catalyst, the activity was higher for $\mathrm{AB}$ than for $\mathrm{SB}$ reaction (table 2, entry 2.e, column 4).

The Arrhenius plot for 50W Co 4.5 (4h) catalyst is shown in Fig. 5.S (as supporting information). The resulting activation energy for $A B$ hydrolysis $\left(E_{a}\right)$ was $(59 \pm 1) \mathrm{kJ} \mathrm{mol}^{-1}$ which is similar to the previously reported for $\mathrm{Co} / \gamma \mathrm{Al}_{2} \mathrm{O}_{3}\left(62 \mathrm{kj} \cdot \mathrm{mol}^{-1}\right)$ [14].

\subsection{Durability upon cycling}

One of the major challenges for the catalysts employed in these hydrogenproducing reactions (1) and (2), is durability upon cycling. This property is an essential requirement for the design of multi-use reactors. For this reason, durability was tested for selected Co catalysts prepared as thin films upon 14 cycles for SB and AB hydrolysis. Results are discussed below.

\subsubsection{Sodium borohydride hydrolysis}

Durability of the $200 \mathrm{~W}$ Co 1.5 (2h) catalyst was tested for SB hydrolysis upon cycling. Figure 7.a shows the plot of the HGR normalized by the initial $\left(\mathrm{HGR}_{0}\right)$ as a function of the cycle number. Catalytic activity is retained during the first two cycles and then catalyst deactivates in cycle 3 . As previously reported, a reactivation step consisting of washing the catalyst with diluted acid $\left(10^{-4} \mathrm{M} \mathrm{HCl}\right)$ was employed after cycle 4 . [20, 31] This reactivation step aims to eliminate the B-O based species accumulated after each use, and has previously shown to be a deactivation mechanism for Co based catalysts for this reaction $[20,31]$. These B-O based species were detected in B1s and O1s levels together with $\mathrm{Na} 1 \mathrm{~s}$ signal from XPS on the used catalyst after cycle 4 (Table 3 ) and by SEM images as a film (Fig 8.b). After diluted acid washing, the catalytic activity was recovered (cycle 5) despite the XPS characteristic signals of B-O species were still present in the catalyst (191.9 eV, Table 3 bonds assigned according to Ref [18] and [37]) and SEM micrographs (Fig 8.c) show partial recovery of the structural features of 
the fresh catalyst (Fig 8.a). Quantification of the XPS spectra permitted to calculate $\mathrm{B} / \mathrm{Co}$ ratio before and after acid washing. Table 3 shows that this ratio is reduced three fold after acid washing (B/Co ratio 0.6 and 0.2 before and after acid washing). This indicates that the $\mathrm{BO}_{2}{ }^{-}$anion adsorbs strongly to the Co surface but small amounts of this anion do not interfere with activity. For the subsequent cycles, dilute acid washing was employed after each cycle. From cycle 5, catalytic activity decreases constantly within the experimental error to reach $76 \%$ of the initial after 14 cycles. This means that dilute acid $\left(10^{-4} \mathrm{M} \mathrm{HCl}\right)$ washing may not be totally efficient to eliminate B-O based species and they finally accumulate and stabilize after each cycle as previously shown [31]. More concentrated acid solutions could not be employed because leaching of the thin film is produced. The acid washing was previously tested at $1,0.1,0.001,10^{-3}$ and $10^{-4} \mathrm{M}$. The latter concentration showed to be the less aggressive for the film. By weighting the supported catalysts before and after each test, no mass loss was found during 14 cycles within the experimental error. However, small losses should be evaluated by analyzing the acid solutions after each washing. These losses could be in part a reason for the deactivation, despite the use of reactivation steps.

\subsubsection{Ammonia borane hydrolysis.}

Durability of the 50WCo 2.8 (4h) catalysts was evaluated for ammonia borane hydrolysis upon cycling. Results are shown in Fig. 7.b. Catalyst deactivates after each run to reach $60 \%$ of its initial activity after 6 cycles. Taking into account reaction (2) one deactivation mechanism could also be the adsorption of $\mathrm{B}-\mathrm{O}$ species as $\mathrm{BO}_{2}{ }^{-}$is the main hydrolysis product together with ammonium (and or ammonia depending the reaction $\mathrm{pH}$ ). For this reason, as well as in section 3.4.1, from cycle 6, after each cycle the catalyst was also washed with dilute acid in order to dissolve adsorbed borates (basic in nature) on Co surface and also ammonia in the form of ammonium cation (pka $\sim 9)$. Figure 7.b shows that in subsequent cycles the catalyst continued to deactivate, indicating no effect of the dilute acid washing. Figure 9 shows SEM micrographs of 50WCo 2.8 (2h) sample after cycle 14 before and after dilute acid washing in comparison with the fresh catalyst. Fig. 9.b shows a film on catalyst surface that might block the access of ammonia borane to the catalyst during reaction. Acid washing was clearly not efficient in eliminating this film as shown in Fig. 9.c. To understand this, the samples where studied by XPS. Table 4 summarizes main results (bonds assigned 
according to Ref [18] and [37]). The persistence of Co- $\mathrm{NH}_{3}$ signals in the $\mathrm{N}$ (1s) level $(\sim 399.6 \mathrm{eV})$ and $\mathrm{Co}-\mathrm{BO}_{2}{ }^{-}$signals in the $\mathrm{B}(1 \mathrm{~s})$ level $(\sim 192 \mathrm{eV})$ for the acid washed catalyst indicates strong Co-adsorbate interactions that cannot be completely eliminated. However, Co- $\mathrm{NH}_{3}$ interaction has shown to be stronger as demonstrated by the comparison of the N/Co ratio obtained by XPS before (0.3) and after (0.2) acidic washing in cycle 6 (Table 4). On the contrary $\mathrm{Co}-\mathrm{BO}_{2}{ }^{-}$interactions were significantly eliminated by the acid washing as shown by quantitative XPS measurements (B/Co ratio 4 before cycle 6 and 0.68 after acid washing). These strong $\mathrm{Co}-\mathrm{NH}_{3}$ interactions have demonstrated to be stable upon acid washing. By weighting supported catalysts before and after each test, no mass loss was found during 14 cycles within the experimental error

\section{Conclusions}

In this paper, supported Co catalysts were prepared for the first time as thin films by magnetron sputtering for $\mathrm{SB}$ and $\mathrm{AB}$ hydrolysis. The preparation technique has shown to be effective in depositing Co catalysts on metallic and polymeric supports which is highly advantageous for technological applications. Co thin films have demonstrated to be stable and well adhered to $\mathrm{Ni}$ foam in the reaction medium. Catalytic activity was improved by varying deposition conditions. Lowering deposition power and increasing deposition pressure was the strategy employed because it produces smaller crystallite size (more amorphous) and smaller column size (more disperse) materials. The highest catalytic activity was obtained for the $50 \mathrm{~W}$ Co 4.5 (4h) sample and resulted in $2650 \mathrm{ml} \cdot \mathrm{min}^{-1} \cdot \mathrm{g}_{\text {catalyst }}{ }^{-1}\left(\mathrm{E}_{\mathrm{a}}=60 \pm 2 \mathrm{~kJ} \cdot \mathrm{mol}^{-1}\right)$ for SB hydrolysis.

Durability of the prepared thin films was also tested for both reactions upon cycling. For SB hydrolysis, the tested catalyst has shown better durability than the one tested for $\mathrm{AB}$ hydrolysis. Basically, for $\mathrm{SB}$ hydrolysis, the adsorption of B-O based species is the main deactivation mechanism which has shown to be in part reversible by acid washing. For $\mathrm{AB}$ hydrolysis, $\mathrm{NH}_{3}$ adsorption on Co surface demonstrated to be stronger than $\mathrm{BO}_{2}{ }^{-}$and resistant to acid washing. 


\section{References}

[1] U.B Demirci, O.Akdim, J. Andrieux, J. Hannauer, R. Chamoun, P.Miele. Fuel Cells, 3 (2010), 335-350. References therein

[2] S.S. Muir, X. Yao. Int J. Hydrogen Energy; 36, (2011), 5983-5997. References therein

[3]Liu, B. H. and Z. P. Li. Journal of Power Sources, 187(2), (2009),527-534. References therein

[4] H.L. Jiang, Q. Xu. Catalysis Today, 170, (2011), 56-63. References therein

[5] U.B. Demirci, P.Miele, Phys. Chem. Chem Phys, 12, (2010); 14665-14651.

References therein

[6] U.B Demirci, O.Akdim, J. Andrieux, J. Hannauer, R. Chamoun, P.Miele, Sci China Chem 53, (2010), 1870-1879. References therein

[7] U. B. Demirci andP. MIELE, Phys. Chem. Chem. Phys., 2014, DOI:

10.1039/C4CP00250D.

[8] Çakanyildirim Ç, Gürü M. Renew Energy, 35, (2010), 839-844.

[9] Shin WH, Yang SH, Choi YJ, Jung HM, Song CO, Kang JK. J Mater Chem, 19, (2009), 4505-4509

[10] Ye W, Zhang H, Xu D, Ma L, Yi B. J Power Sources, 164, (2007), 544-548

[11] N.A. Malvadkar, K. Sekeroglu, W.J. Dressik, M.C. Demirel, J. Power Sources, 196 (2011), 8553-8560

[12] H. Li, J. Liao, X. Zhang, W. Liao, L. Wen, J. Yang, H. Wang and R.Wang, J. Power Sources, 239, (2013), 277-283

[13] T. Umegaki, J.M. Yan, X.B. Zhang, H.Shioyama, N. Kuriyama, Q. Xu, Journal of Power Sources 195, (2010) 8209-8214

[14] Q. Xu, M. Chandra, J. Power Sources 163 (2006) 364-370. 
[15]. Wu, F. Wu, Y. Bai, B. Yi, H. Zhang, Materials Letters, 59, (2005), 1748-1751

[16] H. Tian, Q. Guo, D. Xu, J. Power Sources, 195, (2010), 2136-2142

[17] R. Fernandes, N. Patel, A. Miotello, R. Jaiswal, D.C. Kothari, Int J. H. Energy, 36 (2011) 13379

[18] G.M. Arzac, T.C. Rojas, A.Fernández, Chem. Cat Chem, 3 (2011) 1305-1313.

[19]O. Akdim, R. Chamoun, U.B. Demirci, Y. Zaatar, A. Khoury, P. Miele. Int J H Energy, 36, (2011), 14527-14533.

[20] O.Akdim, U.B. Demirci, P. Miele, Int. J. Hydrogen Energy, 36: 2011; 1366913675.

[21] N. Patel, R. Fernandes, G. Guella, A. Kale, A. Miotello, B. Patton, C. Zanchetta, J. Phys Chem C, 112, (2008), 696 8-6976.

[22] N. Patel, G. Guella, A. Kale, A. Miotello, B. Patton, C. Zanchetta, L. Mirengui, P. Rotolo, Appl. Catal. A: Gen 323 (2007) 18-24.

[23] N. Patel, A. Miotello, V. Bello, Appl. Catal B Environ, 103, (2011), 31-38

[24] N. Patel, R. Fernandes, A. Santini, A. Miotello, Int. J. Hydrogen Energy, 37,2012, $2007-2013$

[25] Nagaraja, H. S. "Journal of superconductivity and novel magnetism, $\mathbf{0 0 0 2 5 ( 0 0 0 0 6 ) : ~}$ (2012) 1901.

[26] V.Godinho, T.C. Rojas, A. Fernández, Microporous and Mesoporous Materials 149, (2012), 142-146.

[27] V.Godinho, C. Lopéz-Santos, T.C. Rojas, D. Philippon, M.C. Jimenez de Haro, S. Lucas, A.Fernandez, Journal of Alloys and Compounds, 536, (2012), S398-S406.

[28] V.Godinho, J.Caballero-Hernández, D.Jamon, T.C.Rojas, R.Schierholz, J.GarcíaLópez, F.J.Ferrer and A.Fernández, Nanotechnology, 24, (2013), 275604 (10pp).

[29]E. Young, D.G. Nocera, V. Bulovic. Energy Environ. Sci., 3, (2010), 1726-1728.

[30] I. Horcas, R. Fernández, J. M. Gómez-Rodríguez, J. Colchero, J. Gómez-Herrero, and A. M. Baro, Rev. Sci. Instrum. 78 , (2007), 013705.

[31] G.M. Arzac, D. Hufschmidt, M.C. Jiménez de Haro, A. Fernández, B. Sarmiento, M.A. Jiménez, M.M. Jiménez. Int. J. Hydrogen Energy, 37, (2012), 14373-14381.

[32] Thornton, J.A., High Rate Thick Film Growth. Annual Review of Materials Science, 7: (1977). p. 239-260. 
[33] G.W. Qin, B. Yang, N. Xiao,Y.P.Ren, M. Jiang, X. Zhao, K. Oikawa. Thin solid films 517 (2009)2984-2987

[34] Sen, B.; Kaufman, C. M. Chem. Soc. Dalton Trans. 64, (1985), 307-313.

[35] J.C Walter, A. Zurawski, D. Montgomery, M. Thornburg, S. Revankar, J. Power Sources, 179 (2008)335-339.

[36] K.A. Holbrook, P.J. Twist, J. Chem. Soc. A, (1971) 890-894.

[37] A.Galtyries, E. Laksono, J.-M Siffre, C. Argile, P. Marcus, Surface and Interface Analysis, 30, (2000), 140-144. 


\section{Acknowledgements}

Financial support from MINECO (CTQ2012-32519), CSIC (PIE 201260E006 and 201060E102), Junta de Andalucía (TEP217, PE2012-TEP862) the EC (CT-REGPOT2011-1-285895, AL-NANOFUNC) is acknowledged. Authors would like to thank to Dr. Carmen López -Santos for the AFM measurements, and to the reviewers for their valuable comments. 


\section{Figure Captions}

Figure 1-a. The $200 \mathrm{~W}$ Co 1.5 (2h) thin film. a.1: Cross sectional SEM images. a.2. Planar SEM image. a.3. TEM image (inset SAED). a.4. AFM image. a.5. Signal intensity profile of image a.4. a. 6 Column size histogram .

Figure 1-b. The 50W Co 1.5 (2h) thin film. b.1: Cross sectional SEM images. b.2. Planar SEM image. b.3. TEM image (inset SAED). b.4. AFM image. b.5. Signal intensity profile of image b.4. b. 6 Column size histogram .

Figure 2- Planar SEM images of 200W Co 1.5 (2h). (a),(d) Si supported; (b),(e) Ni foam supported; (c),(f) PTFE membrane supported. ( a,b,c and d,e,f same scale bar respectively)

Figure 3. XRD measurements performed on (a) 200W Co 1.5 (2h)/ PTFE; (b) 50W Co $1.5(2 \mathrm{~h}) / \mathrm{PTFE}$ thin films.

Figure 4- Planar SEM images of (a) 200W Co 1.5 (2h) (b) 200W Co 1.5 (4h) (c)Cross sectional SEM image of (b)

Figure 5. a. Cross sectional and planar SEM images of the 50W Co (4h) thin films as a function of deposition pressure

Figure 5. b. Catalytic activity and average mesocolumn size as a function of deposition pressure and XRD difractograms for the 50W Co (4h) thin films.

Figure 6- (a) Hydrogen evolution curves for the $50 \mathrm{~W}$ Co 3.2 (4h) catalyzed AB hydrolysis. (b) Hydrogen generation rate as a function of the mass of catalyst for the same catalyst.

Figure 7- Durability of the catalysts upon cycling. Retained activity as a function of the cycle number $\left(\mathrm{HGR} / \mathrm{HGR}_{0}\right.$ ) (a) for SB hydrolysis (b) for $\mathrm{AB}$ hydrolysis. 
Figure 8- Planar SEM images for the cycling experiments for $200 \mathrm{~W}$ Co 1.5 (2h) catalyzed SB hydrolysis (a) Fresh catalyst (b) after 4 cycles (c) after 4 cycles washed with diluted acid.

Figure 9- Planar SEM images for the cycling experiments for 50W Co 2.8 (4h) catalyzed AB hydrolysis (a)Fresh catalyst (b) after 14 cycles (c) after 14 cycles washed with diluted acid

\section{Table legends}

Table 1: Characterization of the Co thin films obtained by magnetron sputtering at 1.5. $10^{-2} \mathrm{mBar}$.

Table 2: Catalytic activity $\left(\mathrm{ml} \cdot \mathrm{min}^{-1} \cdot \mathrm{g}_{\text {catalyst }}{ }^{-1}\right)$ for SBH hydrolysis for the prepared Ni foam supported Co thin films as a function of deposition conditions.

Table 3- XPS analysis during cycling experiments on $200 \mathrm{~W}$ Co 1.5 (2h) catalyst for sodium borohydride reaction.* Ratios obtained by the quantification of the corresponding levels in the XPS spectra.

Table 4- XPS analysis during cycling experiments on 50WCo 2.8 (4h) catalyst for ammonia borane reaction.* Ratios obtained by the quantification of the corresponding levels in the XPS spectra. 


\begin{tabular}{lcccc}
\hline Sputtering power (W) & $\mathbf{5 0}$ & \multicolumn{2}{c}{$\mathbf{2 0 0}$} \\
\hline Deposition time (h) & 2 & 4 & 2 & 4 \\
Film thickness $(\mathbf{n m})$ & 900 & 2000 & 4100 & 7300 \\
Deposition rate $(\mathbf{n m} / \mathbf{s})$ & 0,12 & 0,13 & 0,56 & 0,51 \\
Temperature achieved during the deposition $\left(\mathbf{~}^{\circ} \mathbf{C}\right)$ & $45-55$ & 50 & $120-145$ & $150-175$ \\
\hline
\end{tabular}

TABLE 1 


\begin{tabular}{|c|c|c|c|c|c|c|}
\hline \multirow{2}{*}{ Entry } & \multirow{2}{*}{ Catalyst } & \multicolumn{5}{|c|}{ Deposition Pressure (10 ${ }^{-2}$ mBar $)$} \\
\cline { 3 - 7 } & & $\mathbf{1 . 5 . 1 0}^{-2}$ & $\mathbf{2 . 1 0}^{-2}$ & $\mathbf{2 . 8 . 1 0 ^ { - 2 }}$ & $\mathbf{3 . 2 . 1 0}^{-2}$ & $\mathbf{4 . 5 . 1 0}^{-2}$ \\
\hline 2.a & 200W Co (2h) & 360 & -- & -- & 1350 & -- \\
\hline 2.b & 200W Co (4h) & 350 & -- & -- & -- & -- \\
\hline 2.c & 100W Co (4h) & 500 & -- & -- & -- & -- \\
\hline 2.d & 50W Co (2h) & 700 & -- & -- & -- & -- \\
\hline 2.e & 50W Co (4h) & 670 & 570 & 1550 & 2050 & 2650 \\
\hline
\end{tabular}

TABLE 2 


\begin{tabular}{|c|c|c|c|}
\hline Signal & Fresh Catalyst & After cycle 4 & $\begin{array}{c}\text { After cycle } 4 \\
\text { Diluted acid washing }\end{array}$ \\
\hline $\begin{array}{c}\mathrm{Co} \\
\left(2 \mathrm{p}_{3 / 2}\right)\end{array}$ & $\begin{array}{c}777.8 / 780.9 \mathrm{eV} \\
(\mathbf{C o} / \mathbf{C o - O}) \\
\end{array}$ & $780.9 \mathrm{eV}$ & $780.9 \mathrm{eV}$ \\
\hline O(1s) & $\begin{array}{c}529.33 / 531.7 \mathrm{eV} \\
\text { (O-Co) }\end{array}$ & $531.3 \mathrm{eV}$ & $531 \mathrm{eV}$ \\
\hline B (1s) & ---------- & $191.9 \mathrm{eV}\left(\mathbf{B O}_{2}^{-}\right)$ & $191.5 \mathrm{eV}$ \\
\hline $\mathrm{Na}(1 \mathrm{~s})$ & --------- & $1071.6 \mathrm{eV}$ (Na-B-O) & ------------- \\
\hline${ }^{*} \mathrm{~B} / \mathrm{Co}$ & $\begin{array}{ll}------- \\
\end{array}$ & 0.608 & 0.2 \\
\hline
\end{tabular}




\begin{tabular}{|cccc|}
\hline Signal & Fresh Catalyst & After cycle 6 & $\begin{array}{c}\text { After cycle 6 } \\
\text { Diluted acid washing }\end{array}$ \\
\hline $\begin{array}{c}\mathbf{C o} \\
\left(\mathbf{2} \mathbf{p}_{\mathbf{3} / \mathbf{2}}\right)\end{array}$ & $\begin{array}{c}777.8 / 780.8 \mathrm{eV} \\
(\mathbf{C o} / \mathbf{C o - O})\end{array}$ & $781.8 \mathrm{eV}$ & $780.7 \mathrm{eV}$ \\
\hline $\mathbf{O} \mathbf{( 1 s )}$ & $\begin{array}{c}529.33 / 531.7 \mathrm{eV} \\
(\mathbf{O}-\mathbf{C o})\end{array}$ & $531.8 \mathrm{eV}$ & $531 \mathrm{eV}$ \\
\hline $\mathbf{B ~ ( 1 s )}$ & --------- & $192.1 \mathrm{eV}\left(\mathbf{B O}_{\mathbf{2}}\right)$ & $191.8 \mathrm{eV}$ \\
\hline $\mathbf{N}(\mathbf{1 s})$ & ----------- & $399.9 \mathrm{eV}\left(\mathbf{H}_{\mathbf{3}} \mathbf{N}-\mathbf{C o}\right)$ & $399.6 \mathrm{eV}$ \\
\hline${ }^{*} \mathbf{B} / \mathbf{C o}$ & ------- & 4 & 0.68 \\
\hline$* \mathbf{N} / \mathbf{C o}$ & ------ & 0.3 & 0.2 \\
\hline
\end{tabular}


Supported Co catalysts for SBH and AB hydrolysis prepared by magnetron sputtering Deposition conditions were varied to improve catalytic activity

Decrease in power and increase pressure produces more amorphous and disperse catalysts

Durability upon cycling was studied for both hydrolysis reactions

Acid reactivation was effective for sodium borohydride but not for ammonia borane 


\section{Figure 1.a 200W Co}
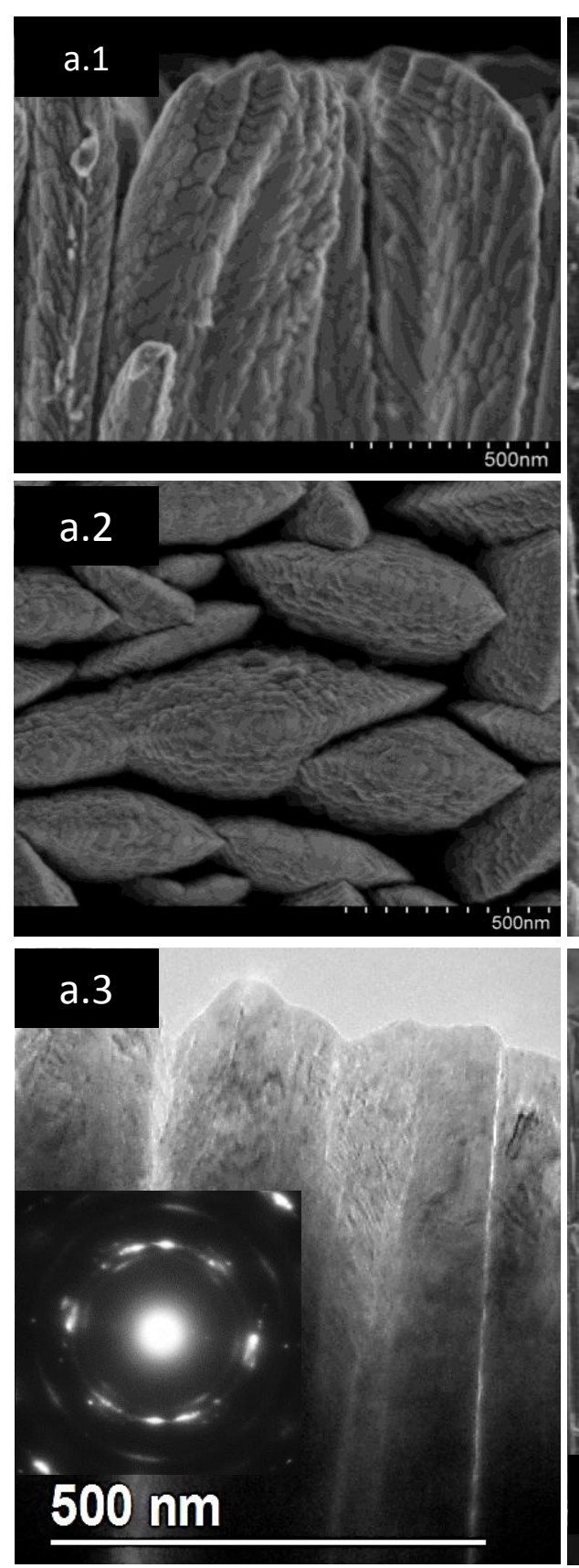

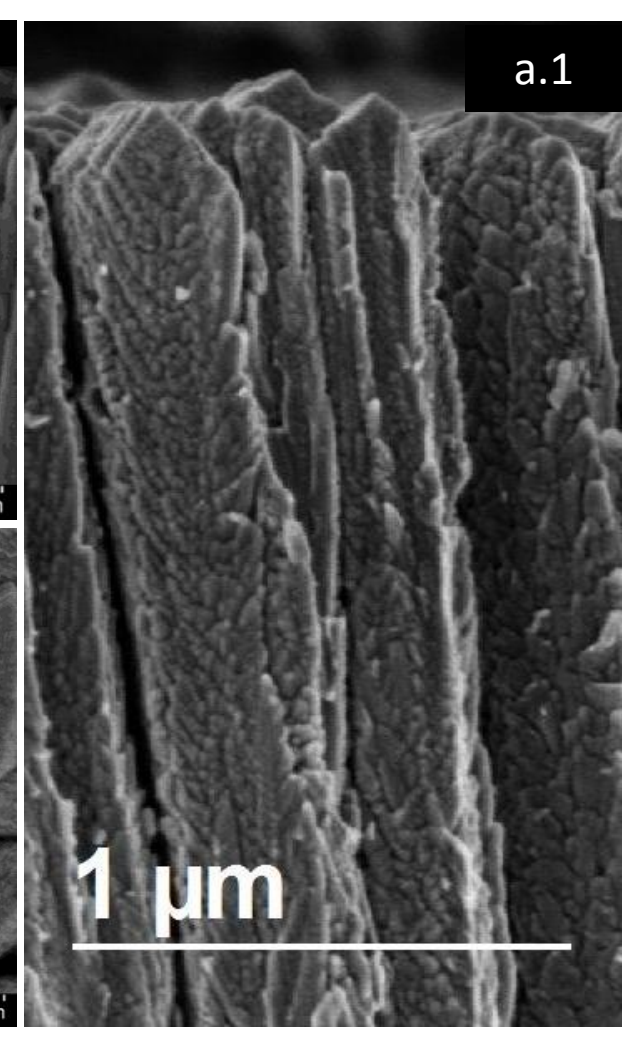

a. 4

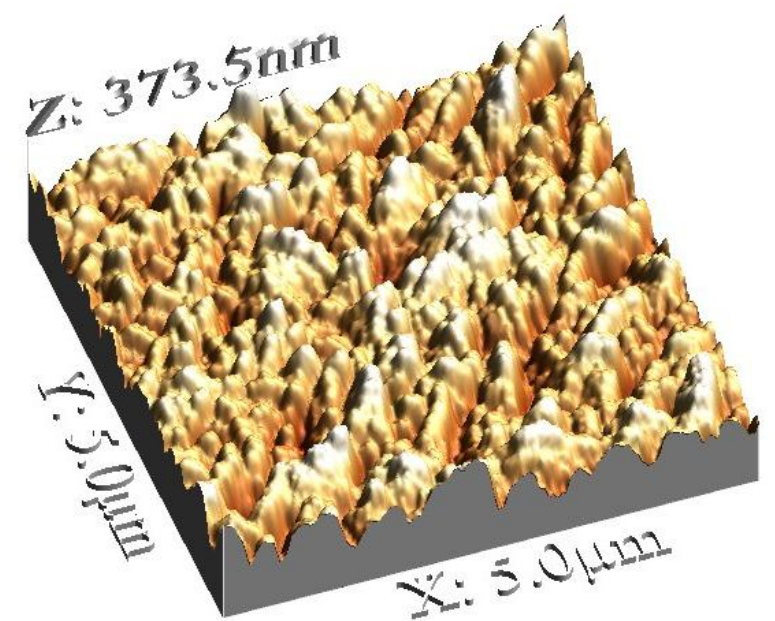

a. 5
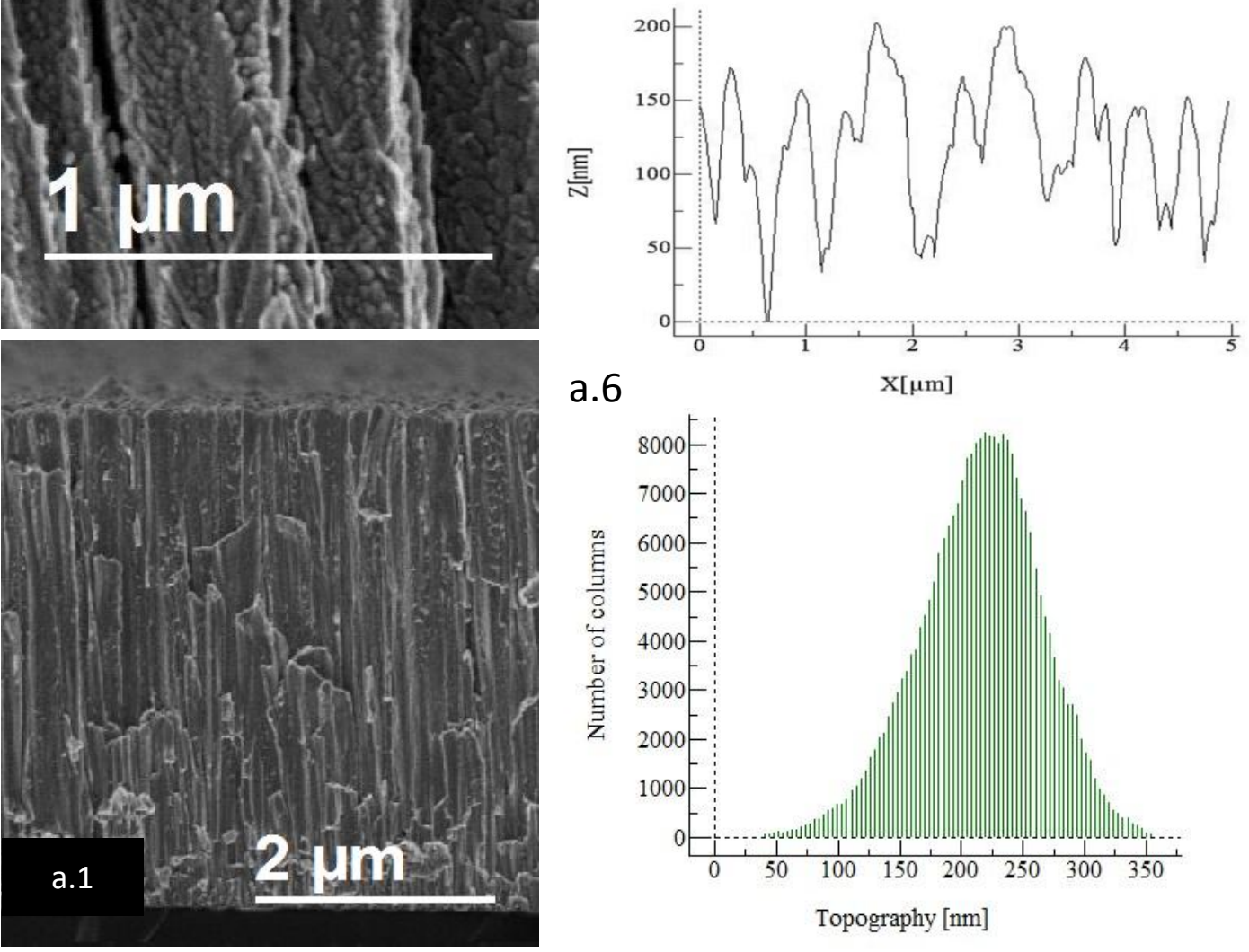

a. 6

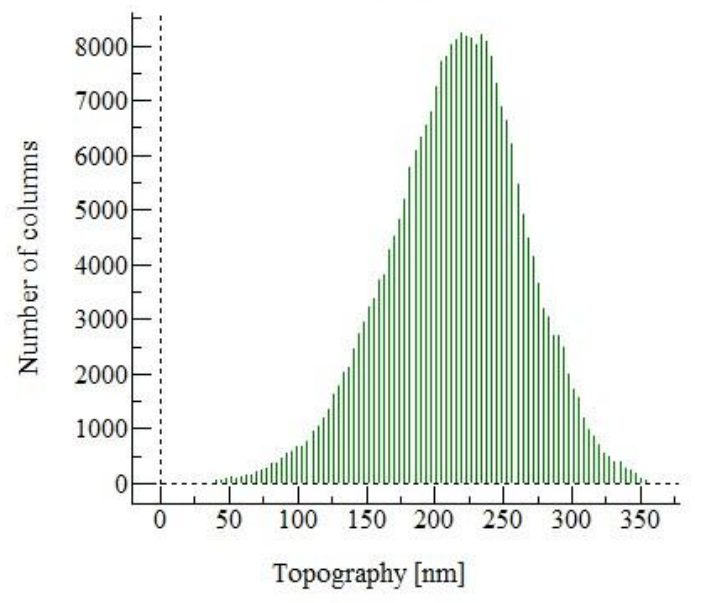


Figure 1.b

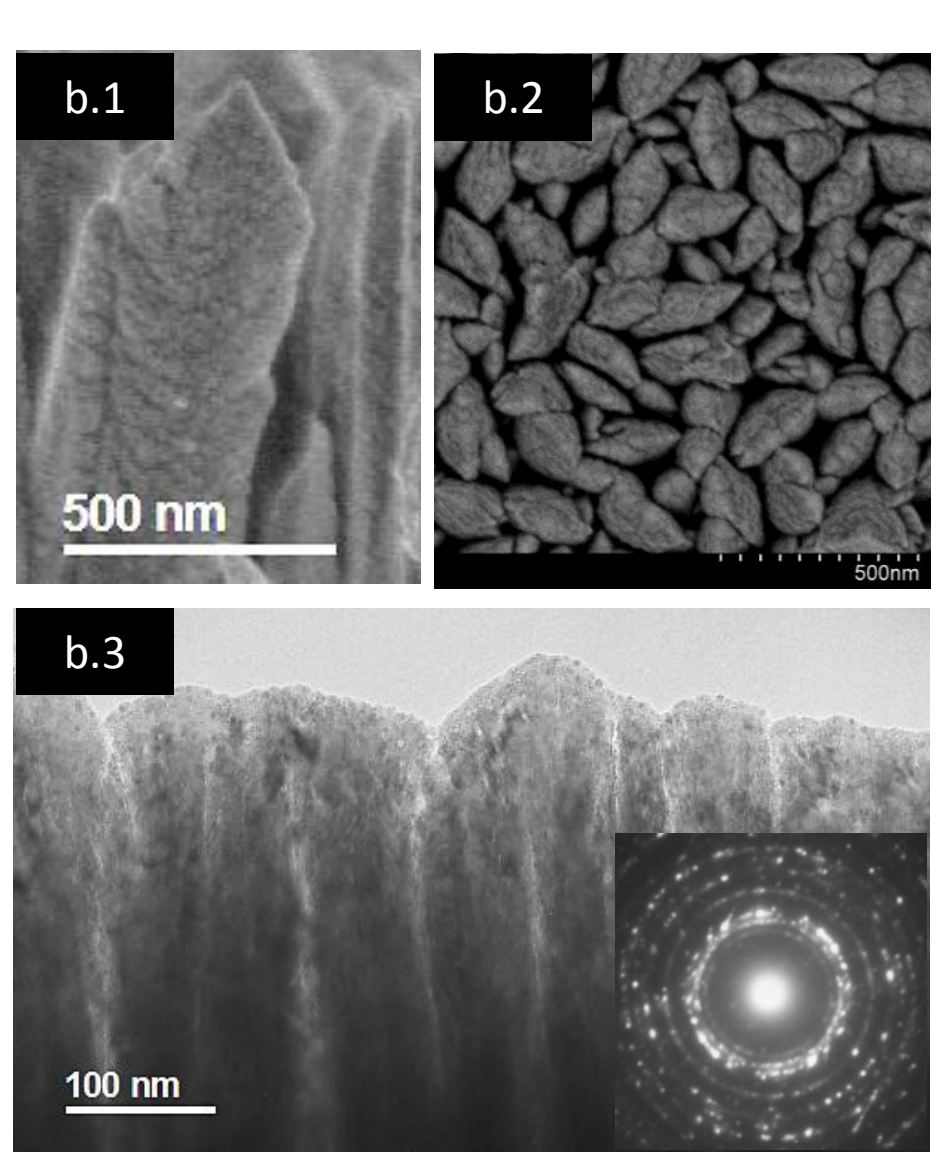

\section{W Co}
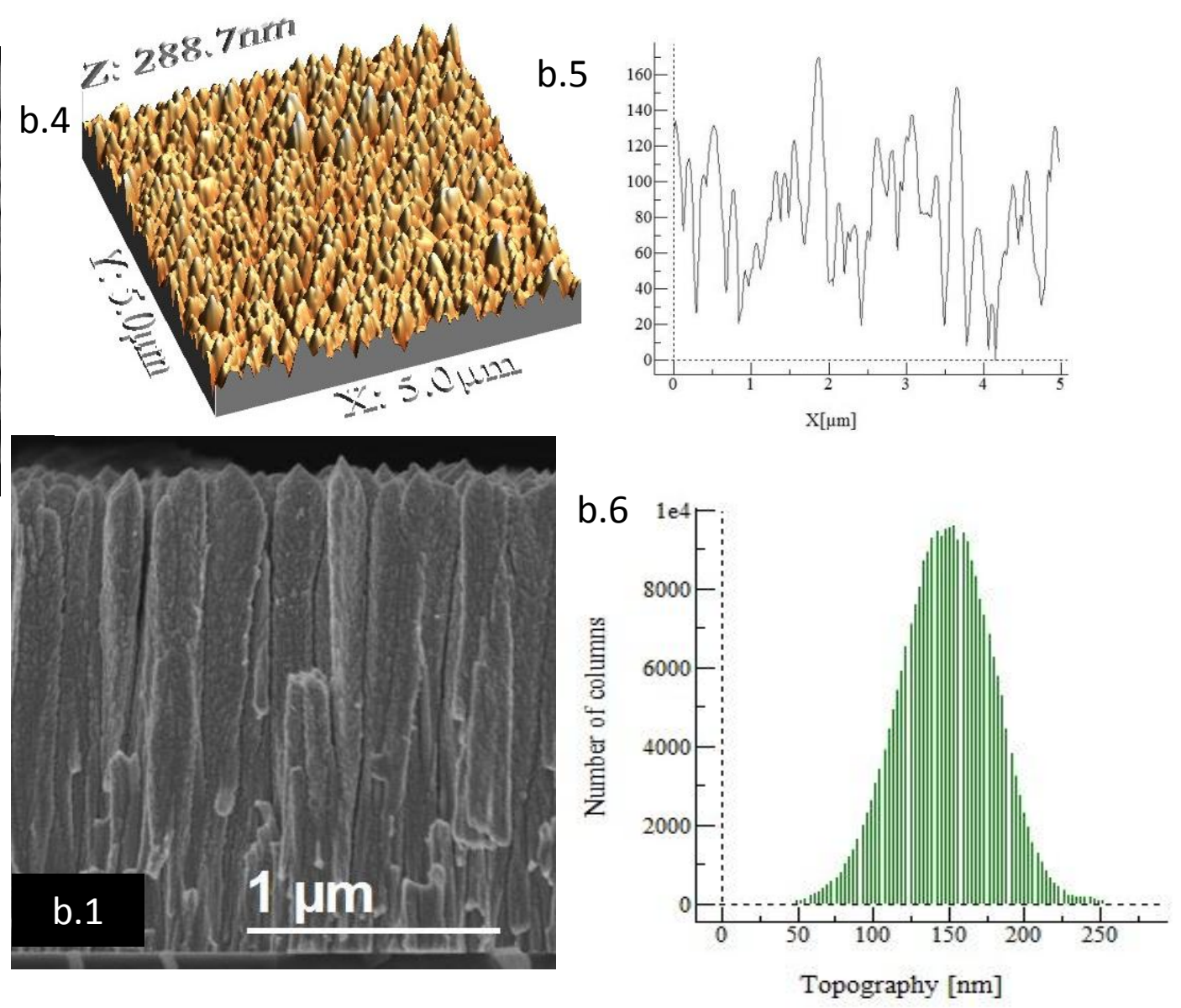
Figure 2

Ni foam

PTFE
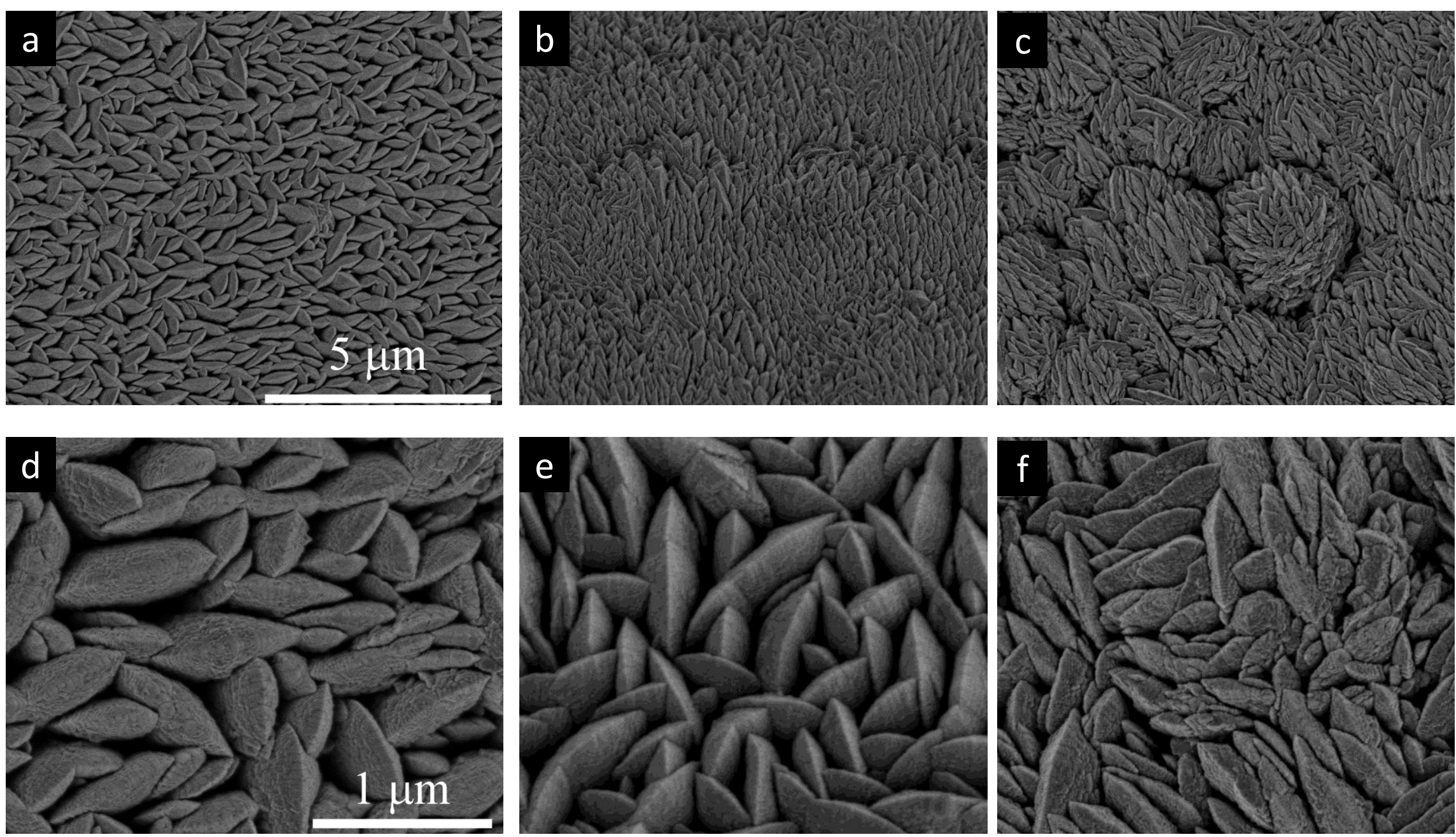
Figure 3

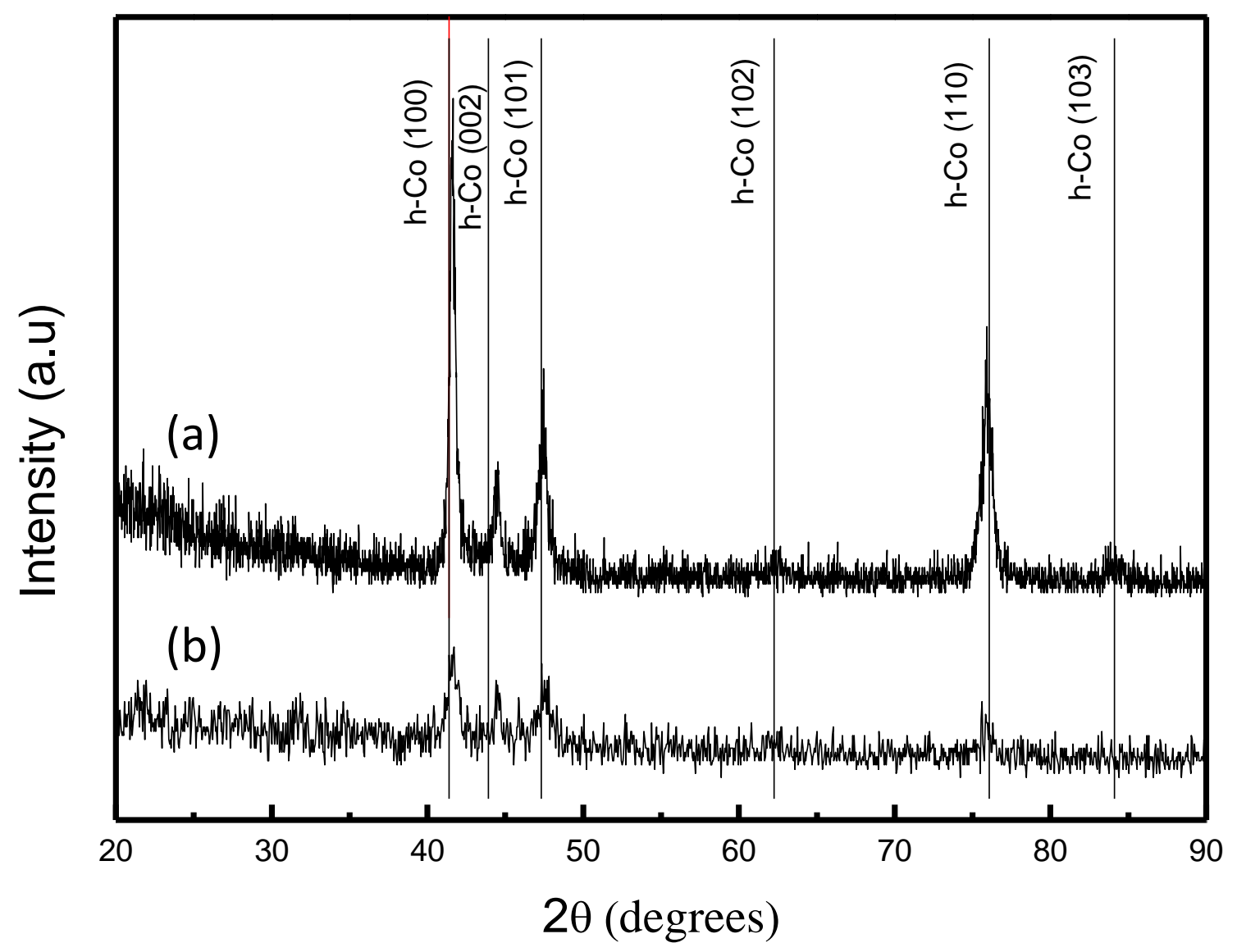


Figure 5.a.

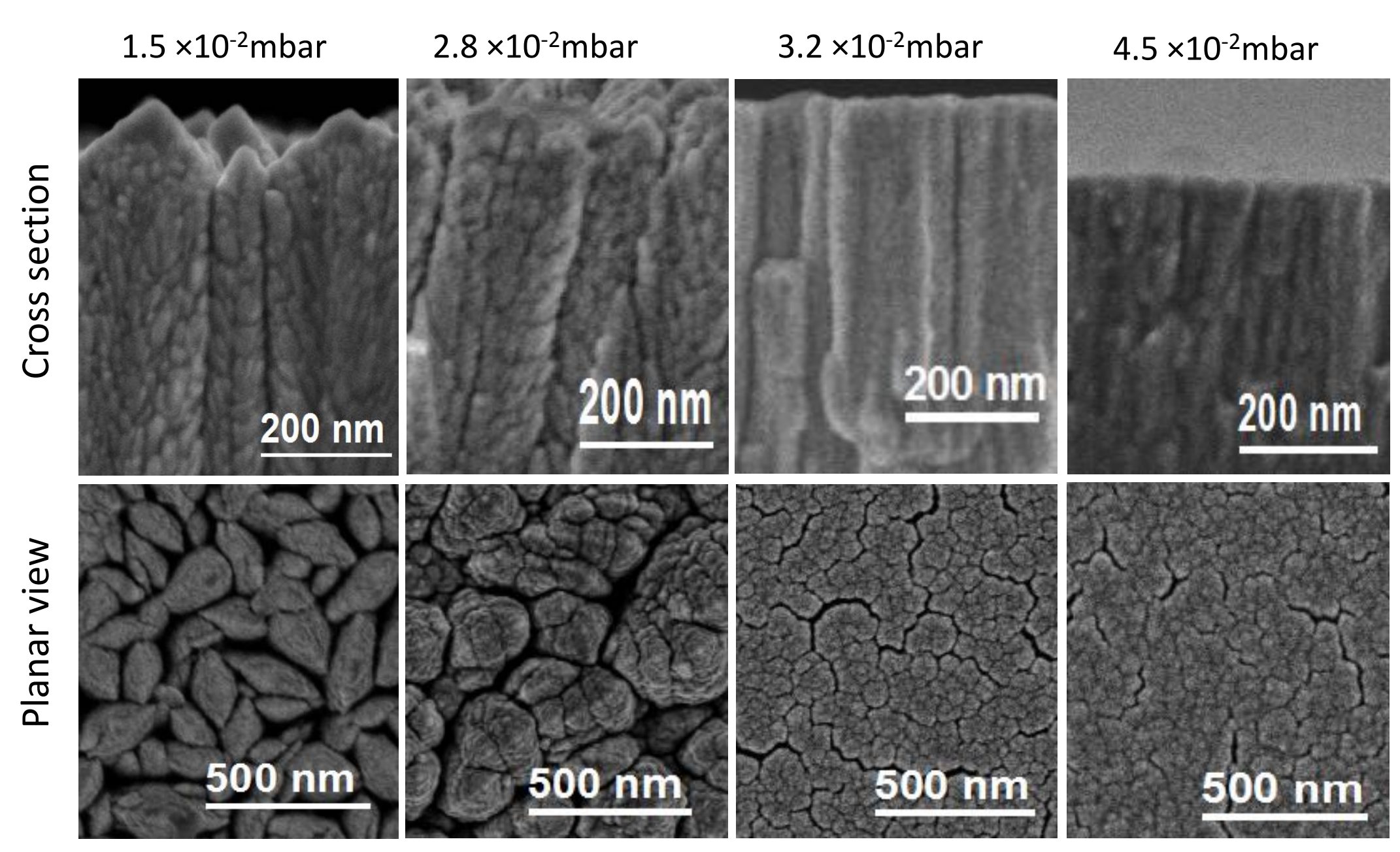


Figure 5.b.
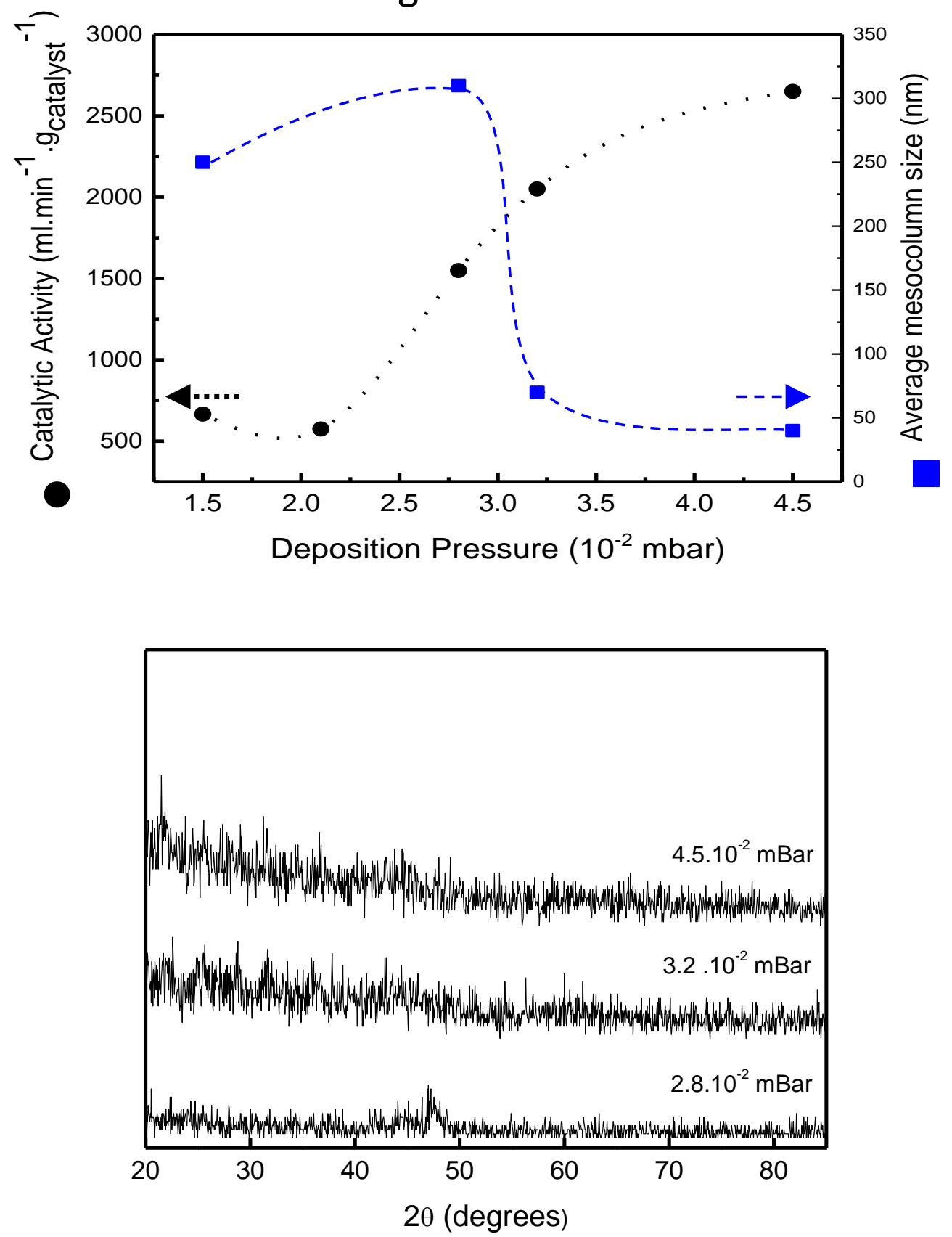
Figure 6
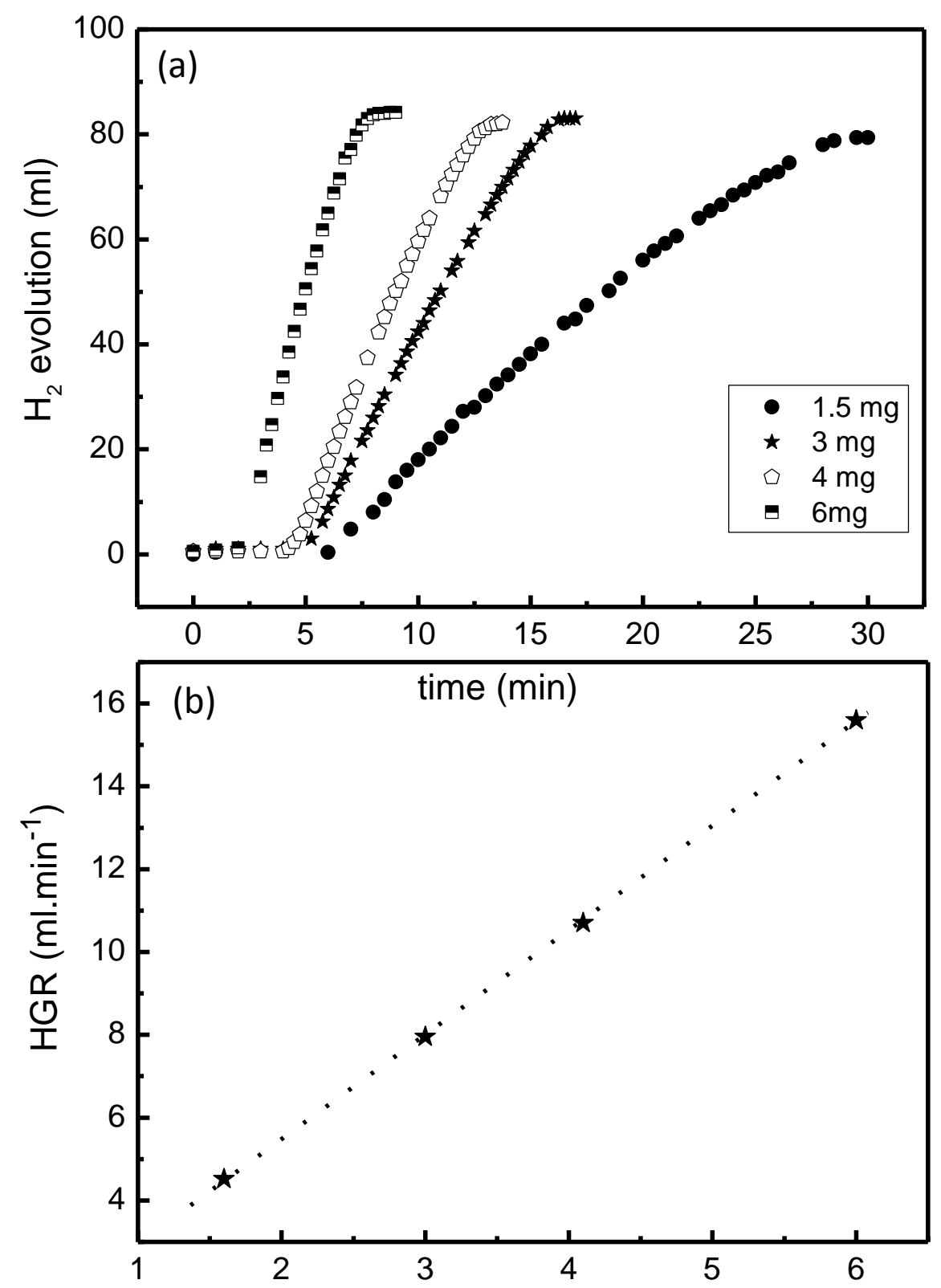
Figure 7
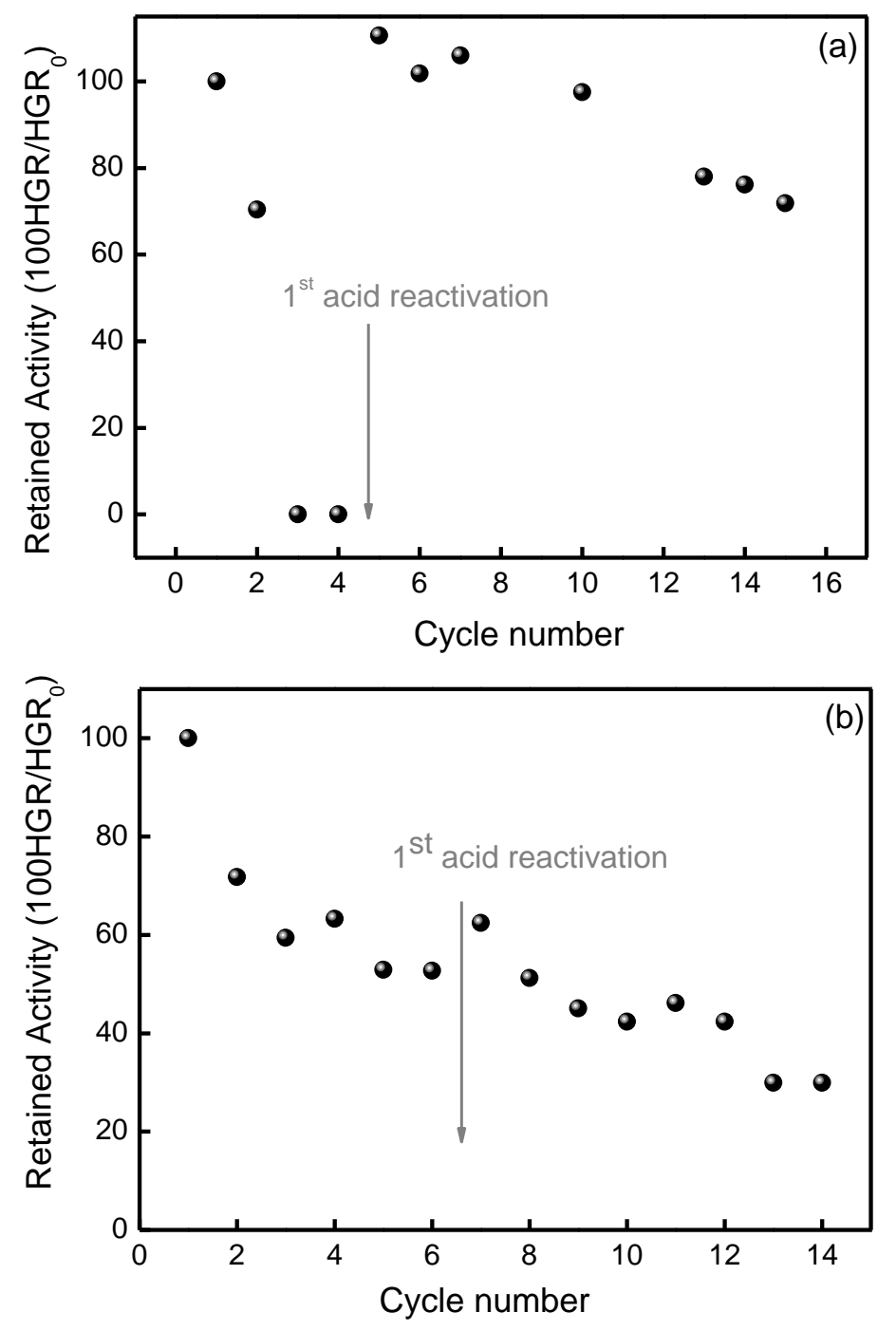

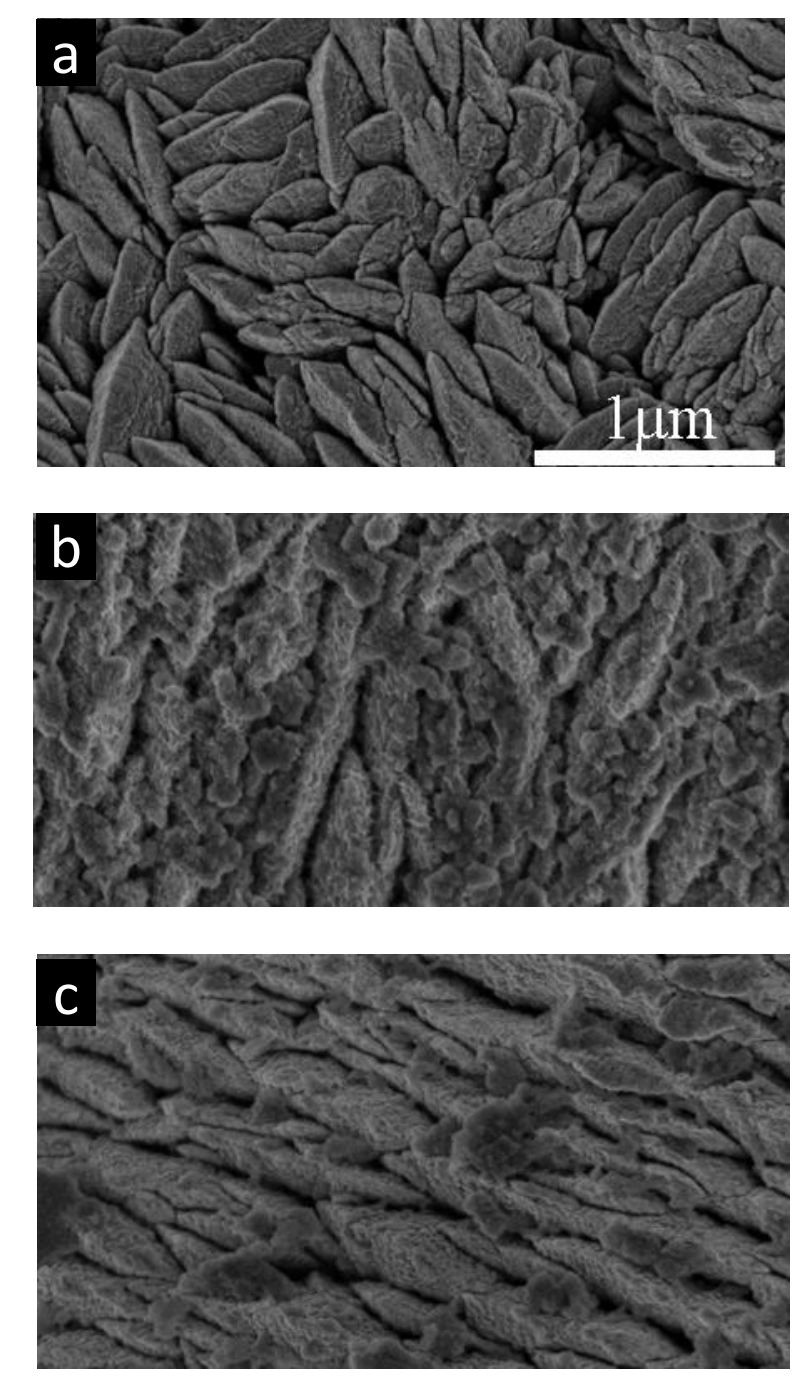

\section{Figure 8}

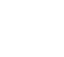

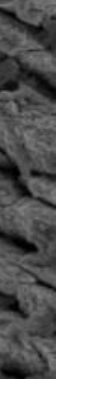

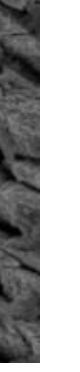


Figure 9
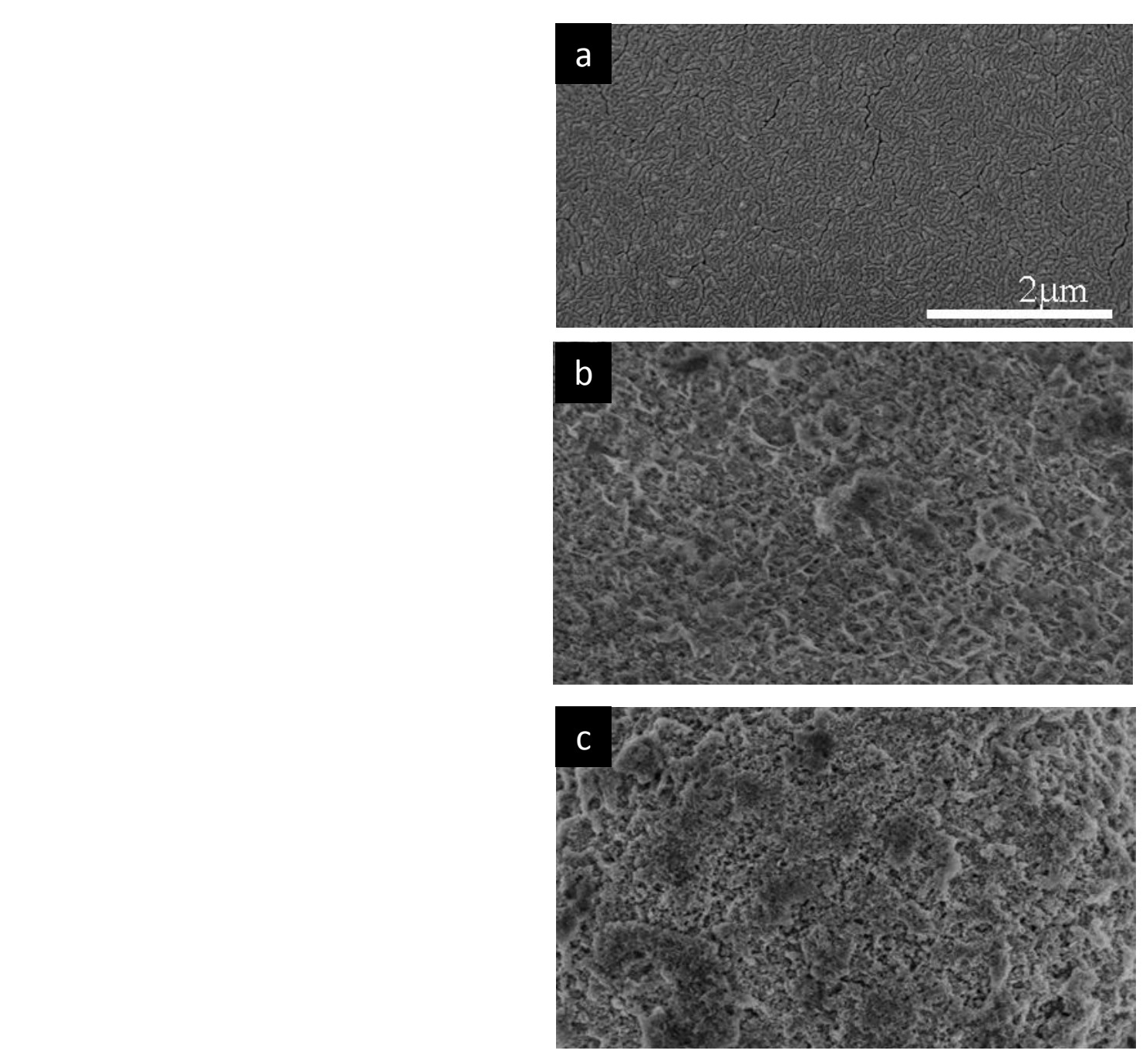

- 


\section{Co thin film}
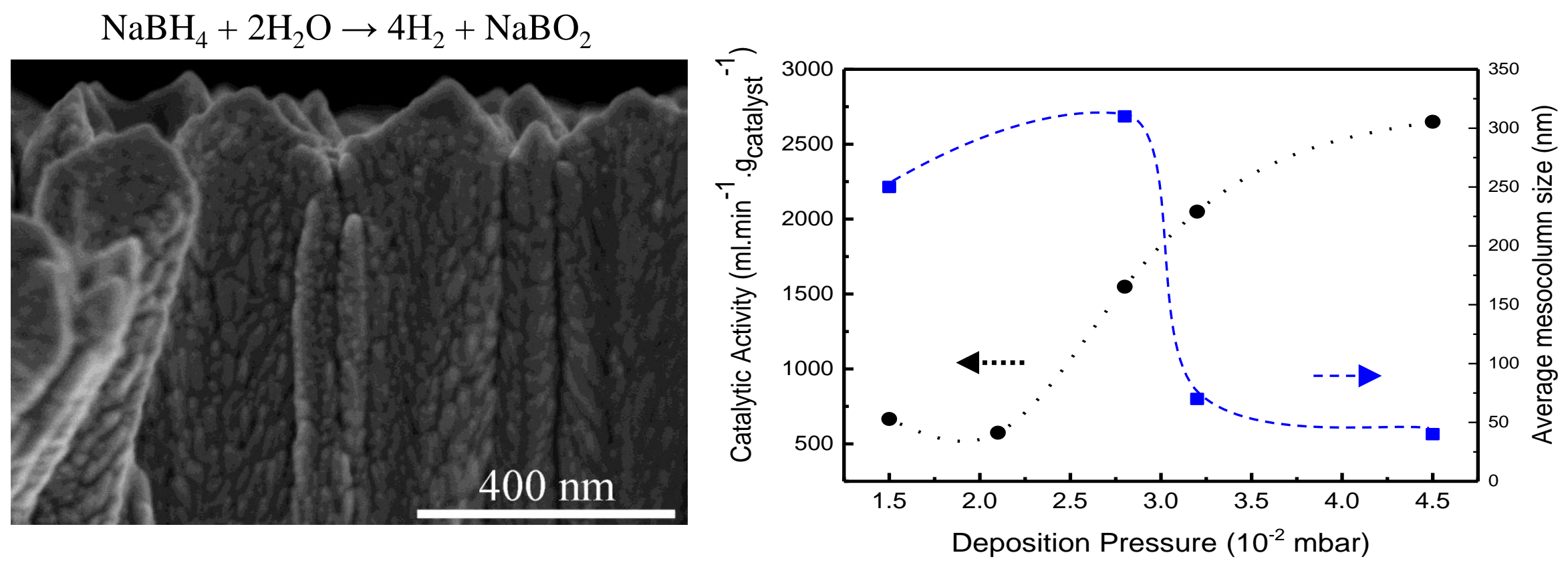